\title{
A Modified STAP Estimator for Superresolution of Multiple Signals
}

\author{
Zhongbao Wang, Junhao Xie, Zilong Ma, and Taifan Quan \\ Department of Electronic Engineering, Harbin Institute of Technology, Harbin 150001, China \\ Correspondence should be addressed to Zhongbao Wang; wzb969@163.com
}

Received 22 January 2013; Accepted 8 April 2013

Academic Editor: Ulrich Nickel

Copyright ( 2013 Zhongbao Wang et al. This is an open access article distributed under the Creative Commons Attribution License, which permits unrestricted use, distribution, and reproduction in any medium, provided the original work is properly cited.

\begin{abstract}
A modified space-time adaptive processing (STAP) estimator is described in this paper. The estimator combines the incremental multiparameter (IMP) algorithm and the existing beam-space preprocessing techniques yielding a computationally cheap algorithm for the superresolution of multiple signals. It is a potential technique for the remote sensing of the ocean currents from the broadened first-order Bragg sea echo spectrum of shipborne high-frequency surface wave radar (HFSWR). Some simulation results and real-data analysis are shown to validate the proposed algorithm.
\end{abstract}

\section{Introduction}

The measurement of the near-surface currents is a very difficult task by using conventional methods, especially under some harsh sea conditions. Many advanced marine measurement instruments, such as drifting buoys [1] and acoustic current meters [2], have been used to collect sea state information. However, it would be very expensive to collect and interpret data from these devices for the sparse spatial sampling provided. Therefore, it is virtually impossible to form the current maps over a widespread ocean surface timely and accurately with these conventional meters.

In recent years, HFSWR has already become a powerful remote-sensing tool which receives increasing attention from oceanographers and research groups for its good ability to determine the large-scale sea state under all weather conditions $[3,4]$. The measurement principle of HFSWR mainly depends on the Bragg resonant scattering theory and Doppler frequency effect theory in the sea echo spectrum of HFSWR. In the absence of ocean currents, the first-order Bragg lines would appear symmetrically above and below the zero Doppler frequency, which are caused by the ocean waves with precisely one-half wavelength of the radar moving towards and away from the radar. In practical application, some displacements will happen in the first-order Bragg lines since the near-surface currents always exist on the ocean [5].
As an extension of shore-based HFSWR, shipborne HFSWR not only inherits all the advantages of shore-based HFSWR but also shows some outstanding features, such as flexibility and mobility. However, some new problems emerge as the radar on board is a moving ship. One of the worst problems is that the first-order Bragg lines have been broadened into two pass bands (when the speed of ship is slow) or one low pass band (when the ship is sailing at a high speed) in the first-order Bragg sea echo spectrum of shipborne HFSWR [6]. For these cases, it will be hard to determine the ocean currents from the broadened sea echo spectrum. Furthermore, the moving ship yields different Doppler shifts to the different azimuth sea echoes. Thus, there exists a certain space-time coupling relation in the received sea echo spectrum of shipborne HFSWR.

The novel space-time IMP algorithm was proposed by Clarke and Spence [7] which was based on one-dimensional IMP algorithm and modified to detect and estimate multiple signals from the conventional beamwidth and (or) Doppler resolution bin. Although space-time IMP estimator effectively improves the robustness of the detection and estimation of multiple signals, the computational load of twodimensional search process is too heavy for real-time application. Lately, Chadwick [8] used the eigendecomposition method instead of the full-search process to reduce the heavy 
computational burden in his proposed polarisation-sensitive IMP algorithm. However, for surface wave radar, this method becomes invalid since the returns of HFSWR are mostly vertical polarization sensitive components.

Many researchers, such as Shaw and Wilkins [9], used beam-space preprocessing technique to reduce the computational load and improve robust performance of highresolution DOA (direction-of-arrival) estimation algorithms. Recently, Hassanien et al. $[10,11]$, proposed a new concept about beam-space preprocessing algorithm which was able to suppress the out-of-sector interferences accompanied with the updated array data. This technique was proven to be more robust than the aforementioned beam-space methods.

In this paper, we combine the space-time IMP algorithm and the adaptive beam-space preprocessing technique yielding a computationally cheap space-time adaptive estimator for detection, estimation and super-resolution of multiple signals. The proposed algorithm is validated by simulation results as well as experimental examples.

This paper is organized as follows. First we introduce the signal model. In the following section, the proposed algorithm and some simulation results are presented. The measurement of the near-surface currents of the ocean by shipborne HFSWR and some real-data analysis are presented in Section 4. The final part is the study conclusion.

\section{Signal Model}

Consider a uniform linear array (ULA) with $M$ omnidirectional antennas and the antenna spacing $d$. If there are $N$ sources that have been received from a far field with different relative delays and attenuations. The received data $\mathbf{X}$ is then given by [7] as follows:

$$
\mathbf{X}=\mathbf{A} \Lambda \mathbf{B}^{H}+\mathbf{N},
$$

where

$$
\begin{gathered}
\mathbf{X}=\left[\begin{array}{llll}
x_{1} & x_{2} & \cdots & x_{M}
\end{array}\right]^{T}, \\
x_{i}=\left[\begin{array}{llll}
x_{i}(1) & x_{i}(2) & \cdots & x_{i}(L)
\end{array}\right],
\end{gathered}
$$

$x_{i}(l), i=1,2, \ldots, M, l=1,2, \ldots, L$ denotes the data received from the $i$ th sensor at $l$ th sampling time, $[\cdot]^{T}$ denotes the transpose operation, $\mathbf{A}=\left[\begin{array}{lllll}\mathbf{a}\left(\varphi_{1}\right) & \mathbf{a}\left(\varphi_{2}\right) & \cdots & \mathbf{a}\left(\varphi_{N}\right)\end{array}\right]^{T}$ is the array manifold matrix,

$$
\mathbf{a}\left(\varphi_{i}\right)=\left[\begin{array}{lll}
1 & \cdots & e^{-j 2 \pi d(M-1) \sin \varphi_{i} / \lambda}
\end{array}\right]^{T}
$$

is the steering vector points to the direction $\varphi_{i}, i=$ $1,2, \ldots, N, \lambda$ denotes wavelength of the radar, $\Lambda$ is a $(N \times N)$ diagonal matrix containing the signal magnitudes, $\mathbf{B}=\left[\begin{array}{llll}\mathbf{b}\left(f_{1}\right) & \mathbf{b}\left(f_{2}\right) & \cdots & \mathbf{b}\left(f_{N}\right)\end{array}\right]$ is a $(L \times N)$ matrix comprising the normalized source waveforms, $\mathbf{b}\left(f_{i}\right)=$ $\left[\begin{array}{llll}1 & e^{-j 2 \pi f_{i}} & \cdots & e^{-j 2 \pi f_{i}(L-1)}\end{array}\right]^{T}$ is the normalized source waveform, $f_{i}, i=1,2, \ldots, N$ is the frequency of $j$ th source, $[\cdot]^{H}$ denotes the Hermitian transpose operation, and $\mathbf{N}$ is the $(M \times L)$ matrix comprising the zero mean and $\sigma^{2}$ variance Gaussian noise.
The $(M \times M)$ covariance matrix of the received data is given by

$$
\mathbf{R}=E\left\{\mathbf{X X}^{H}\right\}=\mathbf{A S} \mathbf{A}^{H}+\sigma^{2} \mathbf{I},
$$

where $E\{\cdot\}$ is the statistical expectation operator, $\mathbf{S}=$ $E\left\{\Lambda \mathbf{B}^{H} \mathbf{B} \Lambda^{H}\right\}$ is the $(L \times L)$ source covariance matrix, and $\mathbf{I}$ is the identity matrix.

\section{Modified STAP Estimator}

3.1. Space-Time IMP. Space-time IMP is a two-dimensional maximum likelihood method which uses a set of spacetime calibration response vectors to match with the received data. Thus, the primary objective of space-time IMP is to maximize the "signal plus noise" to "expected noise" power ratio (SNR). If the maximum output power is over the threshold, then a target is detected and the corresponding space-time calibration response vector is recorded. In order to reduce the sidelobe leakage of the detected targets and improve the detection and estimation of the potential signals in the residual data, the detected targets are removed from the original data through an orthogonal subspace projection matrix before each iterative stage.

According to the definition of SNR in space-time IMP, we have the following [7]:

$$
F(\theta, f)=\frac{\mathbf{W}^{H}(\theta, f) \mathbf{Q} \operatorname{vec}(\mathbf{X}) \operatorname{vec}(\mathbf{X})^{H} \mathbf{Q W}(\theta, f)}{\mathbf{W}^{H}(\theta, f) \mathbf{Q W}(\theta, f)},
$$

where

$$
\mathbf{W}(\theta, f)=\operatorname{vec}\left\{\left(\frac{\mathbf{a}(\theta)}{\mathbf{a}^{H}(\theta) \mathbf{a}(\theta)}\right)\left(\frac{\mathbf{b}(f)}{\mathbf{b}^{H}(f) \mathbf{b}(f)}\right)^{H}\right\}
$$

is the $(M L \times 1)$ space-time calibration response matrix, vec $\{\cdot\}$ is the vectorization operation, and $\mathbf{Q}$ is an $(M L \times M L)$ orthogonal projection matrix, which is given by

$$
\mathbf{Q}=\mathbf{I}-\mathbf{M}\left[\mathbf{M}^{H} \mathbf{M}\right]^{-1} \mathbf{M}^{H}
$$

where $\mathbf{M}$ denotes a sum of space-time calibration response vectors corresponding to the detected signals; that is, $\mathbf{Q}$ projects the received data into a subspace orthogonal to the detected signals.

3.2. Adaptive Beam-Space Preprocessing. The adaptive beamspace preprocessing technique was first mentioned in [10] which used the updated data for adaptive suppression of outof-sector interferences. This technique had been shown to be more robust than the aforementioned beam-space methods.

The primary objective of the data-adaptive beam-space preprocessing is to solve the optimal beam-space matrix 
design problem through minimizing the output power of the transformed data, which can be expressed as follows [12]:

$$
\begin{array}{ll}
\min _{\mathbf{C}} & \operatorname{tr}\left(\mathbf{C}^{H} \mathbf{R} \mathbf{C}\right) \\
\text { subject to } & \mathbf{C}^{H} \mathbf{C}=\mathbf{I} \\
& \mathbf{C}^{H} \mathbf{a}\left(\theta_{b}\right)=1 \quad b=1,2, \ldots, B \\
& \left\|\mathbf{C}^{H} \mathbf{a}\left(\bar{\theta}_{k}\right)\right\| \leq \gamma \quad \bar{\theta}_{k} \in \bar{\Theta}, k=1, \ldots, K,
\end{array}
$$

where $\operatorname{tr}\{\cdot\}$ is the trace of a matrix, $\mathrm{C}$ is the $(M \times B)$ beamspace matrix, $B(B \leq M)$ is the beam-space dimension, $\|\cdot\|$ is the vector 2-norm, $\bar{\Theta}$ denotes all out-of-sector directions which are divided into $K$ angular grids, $\left\{\theta_{b}\right\}_{b=1}^{B}$ and $\left\{\bar{\theta}_{k}\right\}_{k=1}^{K}$ are the angles corresponding to the in-of-sector and out-ofsector directions, and $\gamma$ is the stopband attenuation parameter which should meet the requirement [12]

$$
\begin{array}{ll}
\min _{\mathbf{C}} & \gamma \\
\text { subject to } & \mathbf{C}^{H} \mathbf{C}=\mathbf{I} \\
& \mathbf{C}^{H} \mathbf{a}\left(\theta_{b}\right)=1 \quad b=1,2, \ldots, B \\
& \left\|\mathbf{C}^{H} \mathbf{a}\left(\bar{\theta}_{k}\right)\right\| \leq \gamma \quad \bar{\theta}_{k} \in \bar{\Theta}, k=1, \ldots, K .
\end{array}
$$

After the beam-space transformation, the array steering vector matrix a and manifold matrix $\mathbf{A}$ have already transformed into

$$
\begin{aligned}
\widetilde{\mathbf{a}} & =\mathbf{C}^{H} \mathbf{a}, \\
\widetilde{\mathbf{A}} & =\mathbf{C}^{H} \mathbf{A} .
\end{aligned}
$$

Then, the $(B \times B)$ covariance matrix $\widetilde{\mathbf{R}}$ in beam-space should be rewritten as

$$
\widetilde{\mathbf{R}}=\widetilde{\mathbf{A}} \mathbf{S} \widetilde{\mathbf{A}}^{H}+\sigma^{2} \mathbf{I} .
$$

Obviously, the dimension of the matrix $\widetilde{\mathbf{R}}$ is lower than that of $\mathbf{R}$. This fact is exploited in all beam-space-based methods to reduce computational load compared with the element-space algorithms [10].

3.3. Space-Time Adaptive Beam-Space IMP. In this section, we show how the conventional space-time estimator combines with the adaptive beam-space preprocessing technique to present a computationally cheap space-time adaptive beam-space IMP estimator.

Following the discussion above, the two-dimensional discriminants shown in (5) should be modified as

$$
\widetilde{F}(\theta, f)=\frac{\widetilde{\mathbf{W}}^{H}(\theta, f) \widetilde{\mathbf{Q}} \operatorname{vec}\left(\mathbf{C}^{H} \mathbf{X}\right) \operatorname{vec}\left(\mathbf{C}^{H} \mathbf{X}\right)^{H} \widetilde{\mathbf{Q}} \widetilde{\mathbf{W}}(\theta, f)}{\widetilde{\mathbf{W}}^{H}(\theta, f) \widetilde{\mathbf{Q}} \widetilde{\mathbf{W}}(\theta, f)},
$$

where

$$
\mathbf{W}^{\prime}(\theta, f)=\operatorname{vec}\left\{\left(\frac{\mathbf{C}^{H} \mathbf{a}(\theta)}{\mathbf{a}^{H}(\theta) \mathbf{C C}^{H} \mathbf{a}(\theta)}\right)\left(\frac{\mathbf{b}(f)}{\mathbf{b}^{H}(f) \mathbf{b}(f)}\right)^{H}\right\},
$$

and $\widetilde{\mathbf{Q}}$ has been reduced to a $B L \times B L$ matrix in beam-space domain, which is given by

$$
\widetilde{\mathbf{Q}}=\mathbf{I}-\widetilde{\mathbf{M}}\left[\widetilde{\mathbf{M}}^{H} \widetilde{\mathbf{M}}\right]^{-1} \widetilde{\mathbf{M}}^{H}
$$

where $\widetilde{\mathbf{M}}$ denotes a sum of space-time calibration response vectors corresponding to the detected signals in beam-space domain.

3.4. Threshold Setting. How to select an appropriate threshold to terminate the iterative process in IMP algorithm is very important. Theoretically, when all "significant peaks" have been detected and cleared out from the received data, there is only a completely flat plane in the residual scan [13]. However, it is impossible to accurately estimate the noise statistics from the limited received data. Furthermore, the definition of "significant peak" in IMP algorithm has not been clearly reported.

In the paper, we use a double-threshold setting method to ensure that the iterative process is halted timely. First, we check two successive scans before the next iteration. If the difference between the two scans is comparable to that of the "expected noise" level, that is, no "significant peak" appears during the last scan, then the iterative process should be halted. Besides, if the difference between two successive estimations has reached the preset threshold, which suggests that the iterations are estimating the same target, then the iterative process should be halted as well.

3.5. Simulation Results. Several simulation results are shown in this section to test the performance of the modified algorithm through comparing it with several conventional algorithms.

During the simulations, we assume that the radar works at $f=6 \mathrm{MHz}$, which contains an ULA with $M=8$ omnidirectional sensors and the elements are spaced onehalf wavelength apart. The half power beamwidth is approximately $13^{\circ}$. The number of snapshots $L=32$ and the beamspace dimension $B=2$ are chosen for our simulations. The adaptive beam-space matrix has been solved using the cvx optimization MATLAB toolbox. Since the minimum value in (9) is $\gamma_{\min }=0.0676$, we take the parameter $\gamma=0.07$ for (8). Furthermore, the two simulation targets $\left(0.2 \mathrm{~Hz}, 86^{\circ}\right)$ and $\left(0.3 \mathrm{~Hz}, 90^{\circ}\right)$ are also selected in the simulations.

To define a successful experiment, we use the criterion mentioned in [14] if

$$
\sum_{i=1}^{2}\left|\widehat{\theta}_{i}-\theta_{i}\right|<\left|\theta_{1}-\theta_{2}\right|
$$

where $\widehat{\theta}_{i}$ and $\theta_{i}(i=1,2)$ are, respectively, the estimated and truth values, then the two signals are successfully resolved.

Figures 1 and 2 illustrate the probability of source resolution and their root mean square error (RMSE) versus SNR in Doppler domain, respectively. The conventional space-time IMP, space-time beam-space IMP which combines spacetime IMP with discrete fourier transform (DFT) matrix beam-space processing technique [15], and 64 points and 256 


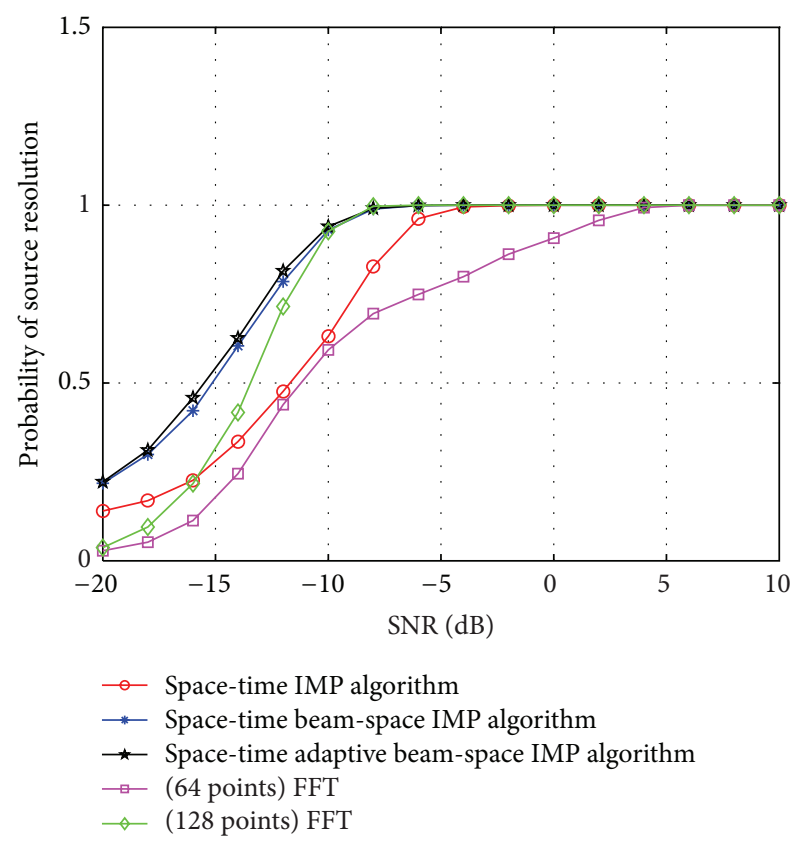

Figure 1: Probability of source resolution versus SNR in Doppler domain.

points FFT results are used for the comparison in the figures. We find that the beam-space-based algorithms show better resolution and smaller RMSE than the other algorithms in resolving the two simulation targets. Thus, it is reasonable to conclude that the beam-space-based methods require less observation time but maintain high Doppler accuracy compared with the conventional algorithms. By the way, all the simulation results shown in this section have averaged over 1000 independent Monte Carlo experiments.

Figures 3 and 4 are the probability of source resolution and their RMSE versus SNR in azimuth domain, respectively. According to these figures, the beam-space-based algorithms show better performance than the other algorithms. Comparing these methods, the space-time adaptive beam-space IMP algorithm shows the highest robust and lowest RMSE in resolving the two simulation targets. Thus, it is reasonable to conclude that the proposed algorithm requires smaller antenna array and lower SNR threshold for detection and estimation of multiple signals when compared with the conventional DOA algorithms.

\section{Shipborne HFSWR}

4.1. Space-Time Coupling Relation. In [6], Xie et al. had proven that the first-order Bragg spectrums were broadened along the azimuthal directions from the real-data analysis. The authors concluded that there was a space-time coupling relation existed in the first-order Bragg sea echo spectrum of shipborne HFSWR.

Assuming that both the transmitting and the receiving antennas of HFSWR are mounted on a ship which is moving in the positive direction of the $x$-axis at a constant speed $v_{s}(\mathrm{~m} / \mathrm{s})$, as shown in Figure 5.

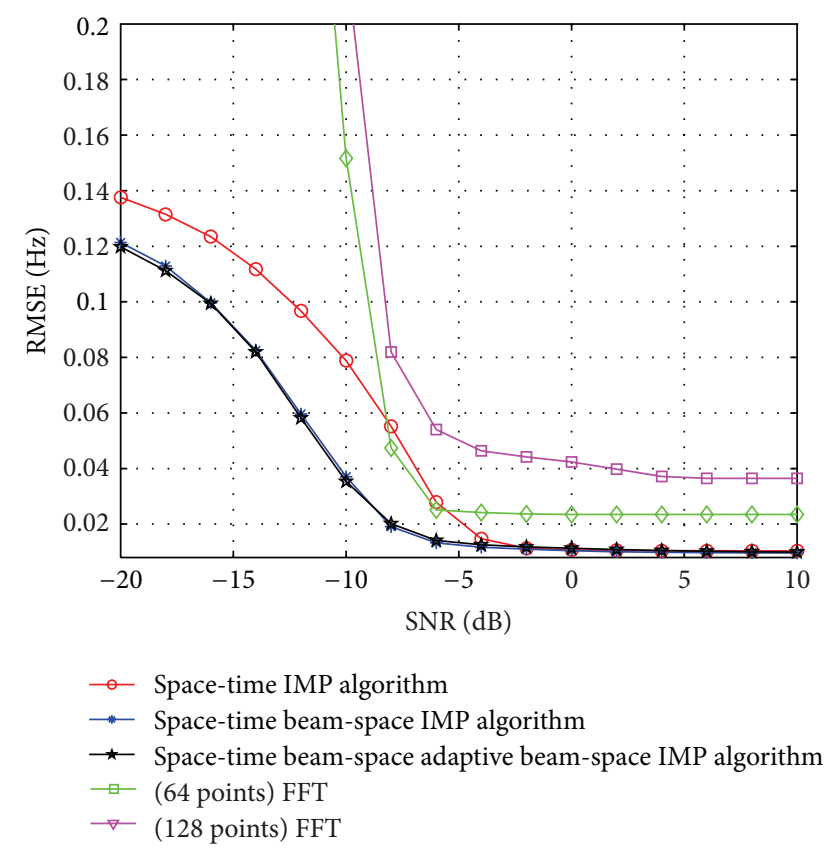

Figure 2: Doppler estimated RMSE versus SNR.

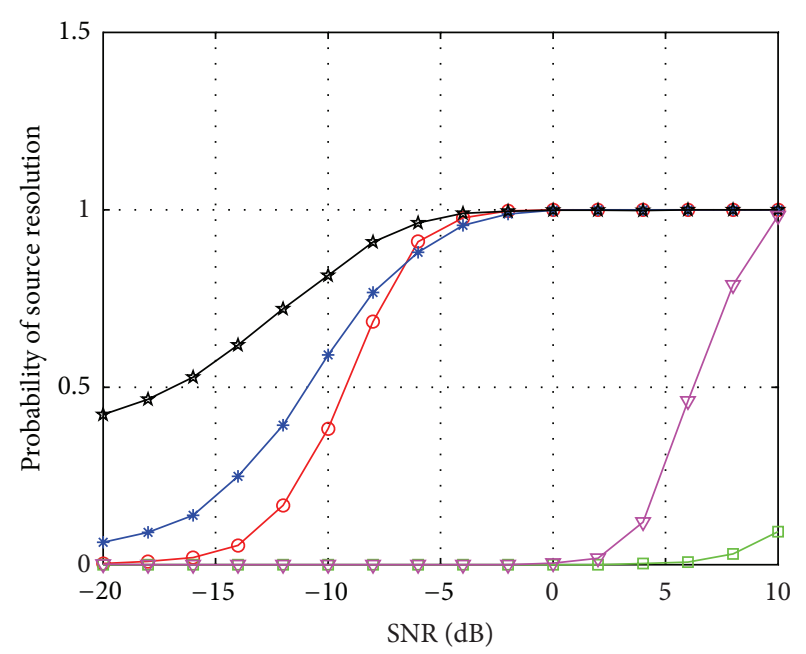

$\rightarrow$ Space-time IMP algorithm

$\rightarrow$ - Space-time beam-space IMP algorithm

* Space-time beam-space adaptive beam-space IMP algorithm

$\because-$ (32 snapshots) MUSIC algorithm

$\rightarrow$ (256 snapshots) MUSIC algorithm

FIGURE 3: Probability of source resolution versus SNR in azimuth domain.

In the absence of ocean current, the space-time coupling relation in the first-order Bragg sea echo spectrum of shipborne HFSWR can be expressed as follows [16]:

$$
f_{d}=\frac{2 v_{s}}{\lambda} \cos \phi+f_{B},
$$

where $\phi \in[0, \pi]$ is the azimuth direction, $f_{B}= \pm \sqrt{g / \pi \lambda}$ are the first-order Bragg frequencies, the positive and negative 


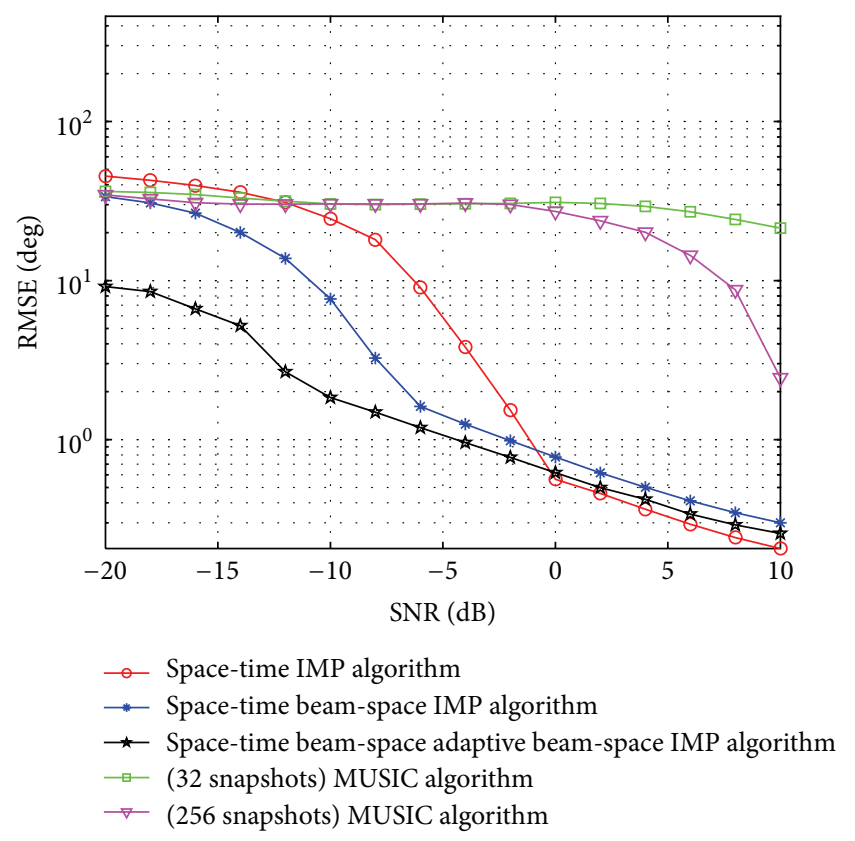

FIGURE 4: Azimuth estimated RMSE versus SNR.

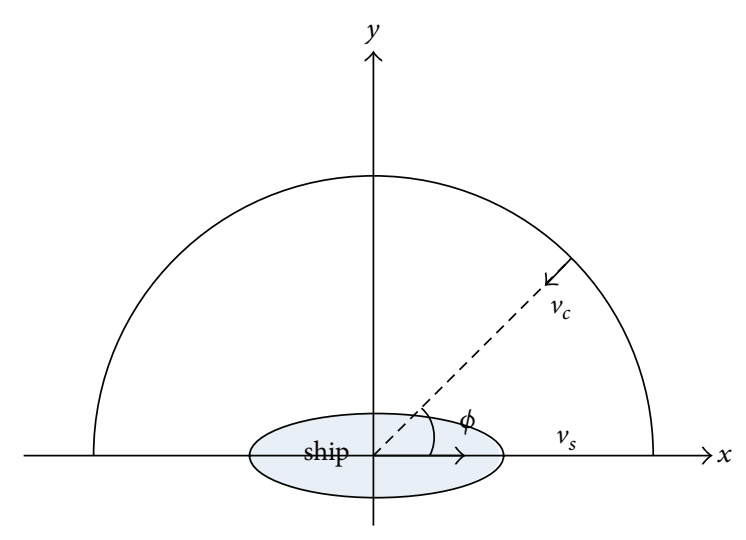

FIGURE 5: Schematic of shipborne HFSWR and current vector.

signs are, respectively, the Bragg waves moving towards and away from the radar.

4.2. Current Measurement. In the presence of ocean currents, the first-order Bragg lines in (16) are shifted from the theoretical positions. The displacements are proportional to the radial current velocities. Thus, (16) should be rewritten as [17]

$$
f_{d}=\frac{2 v_{s}}{\lambda} \cos \phi+f_{B}+\frac{2 v_{c}(\phi)}{\lambda} .
$$

As shown in (17), the first-order Bragg lines are related to the azimuth directions as well as the speed of ship and currents. Therefore, the first-order Bragg peaks are not only displaced, but also broadened into two pass bands (for slow ship speed case) in the first-order Bragg sea echo spectrum of shipborne HFSWR.

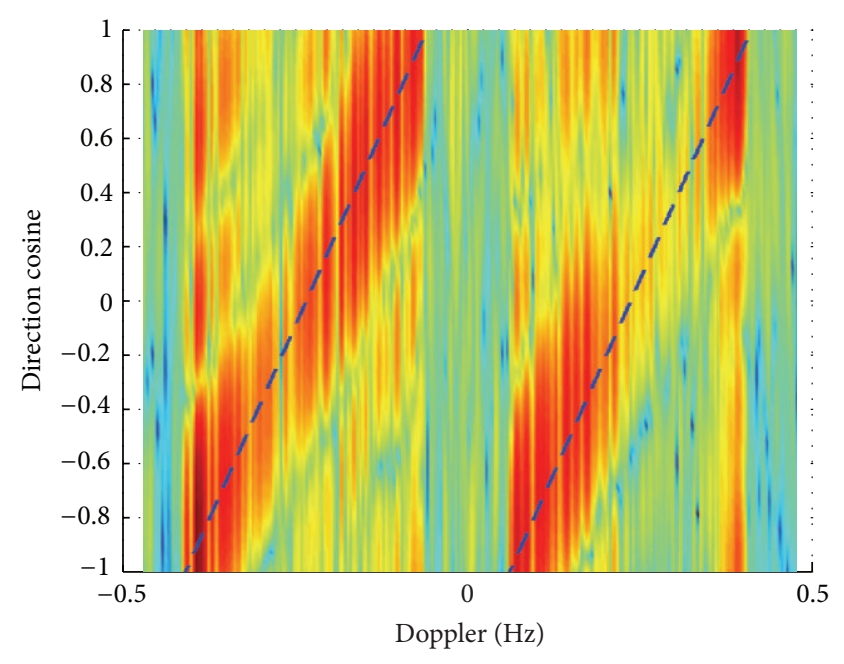

FIGURE 6: Space-time spectrum of real data processed by DFT and DBF cascaded processing. -- denotes the theoretical value $\left(f_{0}=\right.$ $\left.5.283 \mathrm{MHz}, T_{p}=0.262 \mathrm{~s}, d=14 \mathrm{~m}\right)$.

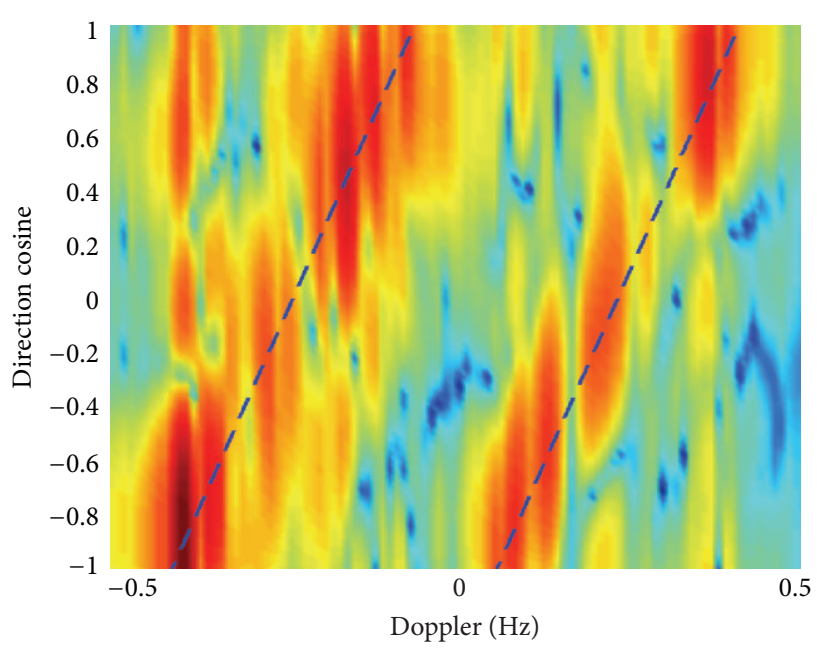

FIGURE 7: Space-time spectrum of real data processed by IMP.

4.3. Real-Data Analysis. The real data used in this paper was received from the shipborne HFSWR experiments conducted on the Yellow Sea of China on September 8, 1998 [6]. Figures 6 and 7 show the space-time coupling relation in the sixth range bin of the real-data file 1128 (containing 7 channels $\times$ 256 samples $\times 32$ range bins and the ship speed was about $4.8 \mathrm{~m} / \mathrm{s}$ ), which is processed through the DFT plus DBF (Digital Beamforming) cascade processing and the proposed algorithm. As shown in the figures, the first-order Bragg lines are broadened along the azimuth directions, which tally well with the theoretical lines in (16). The displacements may be caused by ocean currents or interferences.

Table 1 is an example of the DOA and Doppler estimations. The modified STAP estimator is used to process the above-mentioned real data within the section between the azimuth $40^{\circ}$ and $90^{\circ}$. Since there was no information about the sea state recorded during the experiment, we 


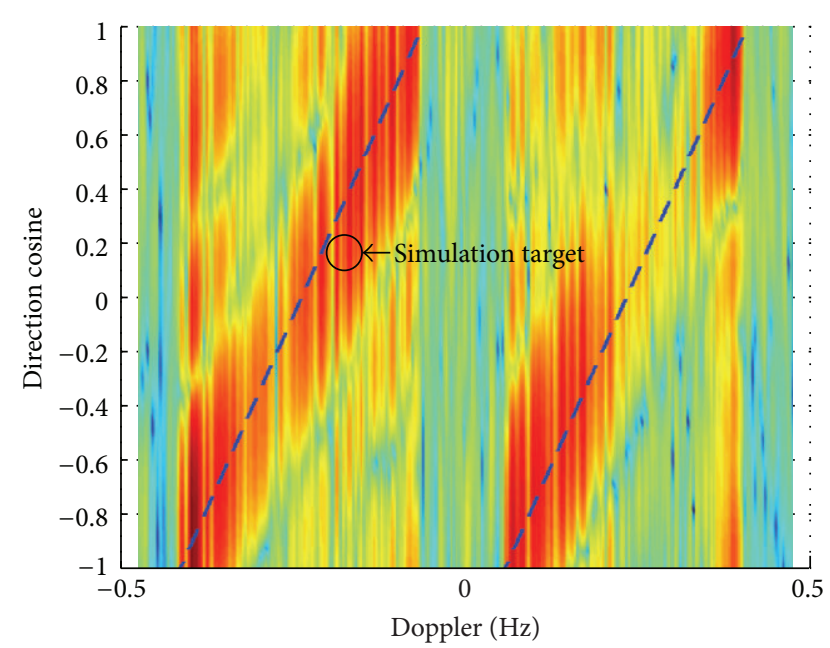

FIgURE 8: Space-time spectrum of real data adds simulation target.

TABLE 1: DOA and Doppler estimations.

\begin{tabular}{lccccc}
\hline Target & 1 & 2 & 3 & 4 & 5 \\
\hline DOA (Deg) & 62.72 & 50.12 & 64.06 & 52.56 & 63.35 \\
Doppler (Hz) & -0.1582 & -0.1154 & -0.1596 & -0.1292 & -0.1589 \\
Theoretical (Hz) & -0.1541 & -0.1220 & -0.1577 & -0.1278 & -0.1558 \\
Velocity (m/s) & 0.1166 & -0.1869 & 0.0519 & 0.0398 & 0.0885 \\
\hline
\end{tabular}

TABLE 2: DOA and Doppler estimations.

\begin{tabular}{lccccc}
\hline Target & 1 & 2 & 3 & 4 & 5 \\
\hline DOA (Deg) & 82.97 & 67.33 & 64.19 & 81.97 & 52.56 \\
Doppler (Hz) & -0.1833 & -0.1627 & -0.1567 & -0.1825 & -0.1176 \\
Theoretical (Hz) & -0.2130 & -0.1668 & -0.1581 & -0.2100 & -0.1238 \\
\hline
\end{tabular}

here assume that the detected targets near the theoretical first-order Bragg lines are considered as ocean currents and their displacements are proportional to their radial velocities. Based on this assumption, five currents have been detected and estimated. All of them are very close to the theoretical first-order Bragg frequencies and their corresponding radial velocities are calculated in the table.

Table 2 is another example for better understanding the robustness of the proposed algorithm, where we add a simulation target $\left(-0.18 \mathrm{~Hz}, 80^{\circ}\right)$ to the real data used previously, as shown in Figure 8. The added simulation target is detected correctly and the estimations of DOA and Doppler frequency are within $2^{\circ}$ and $0.0025 \mathrm{~Hz}$ of the true signal position.

\section{Conclusion}

In this paper, we have introduced a modified space-time adaptive processing estimator that can be used for the detection, estimation, and superresolution of multiple signals. The method combines the conventional IMP method and the existing adaptive beam-space preprocessing techniques yielding a computationally cheap algorithm for estimating the near-surface currents of the ocean from the broadening of the first-order Bragg sea echo spectrum of shipborne HFSWR. The proposed algorithm is validated by simulation results as well as experimental examples.

\section{Acknowledgment}

This work is supported by the State Key Program of National Natural Science of China (Grant no. 61132005).

\section{References}

[1] S. Tyrberg, O. Svensson, V. Kurupath, J. Engström, E. Strömstedt, and M. Leijon, "Wave buoy and translator motionson-site measurements and simulations," IEEE Journal of Oceanic Engineering, vol. 36, no. 3, pp. 377-385, 2011.

[2] F. J. Bugnon and I. A. Whitehouse, "Acoustic doppler current meter," IEEE Journal of Oceanic Engineering, vol. 16, no. 3, pp. 420-426, 1991.

[3] L. Wyatt, "Wave mapping with HF radar," in Proceedings of the 10th IEEE/OES Working Conference on Current, Waves and Turbulence Measurement (CWTM '11), pp. 25-30, Monterey, Calif, USA, March 2011.

[4] J. S. Bathgate, M. L. Heron, and A. Prytz, "A method of swell-wave parameter extraction from HF ocean surface radar spectra," IEEE Journal of Oceanic Engineering, vol. 31, no. 4, pp. 812-818, 2006.

[5] B. J. Lipa and D. E. Barrick, "Least-squares methods for the extraction of surface currents from CODAR crossed-loop data: application at ARSLOE," IEEE Journal of Oceanic Engineering, vol. 8, no. 4, pp. 226-253, 1983.

[6] J. Xie, Y. Yuan, and Y. Liu, "Experimental analysis of sea clutter in shipborne HFSWR," IEE Proceedings: Radar, Sonar and Navigation, vol. 148, no. 2, pp. 67-71, 2001.

[7] I. J. Clarke and G. Spence, "A space-time estimator for the detection and estimation of multiple sinusoidal signals," in Proceedings of the IEE Colloquium High Resolution Radar and Sonar (Ref. No. 1999/051), pp. 9/1-9/6, London, UK, 1999.

[8] A. Chadwick, "Superresolution for high-frequency radar," IET Radar, Sonar and Navigation, vol. 1, no. 6, pp. 431-436, 2007.

[9] A. Shaw and N. Wilkins, "Frequency invariant electro-magnetic source location using true time delay beam space processing," in Proceedings of the 4th IEEE International Symposium on Phased Array Systems and Technology (Array'10), pp. 998-1003, Boston, Mass, USA, October 2010.

[10] A. Hassanien, S. A. Elkader, A. B. Gershman, and K. M. Wong, "Convex optimization based beam-space preprocessing with improved robustness against out-of-sector sources," IEEE Transactions on Signal Processing, vol. 54, no. 5, pp. 1587-1595, 2006.

[11] A. Hassanien and S. A. Vorobyov, "A robust adaptive dimension reduction technique with application to array processing," IEEE Signal Processing Letters, vol. 16, no. 1, pp. 22-25, 2009.

[12] A. Hassanien and S. A. Vorobyov, "New results on robust adaptive beamspace preprocessing," in Proceedings of the 5th IEEE Sensor Array and Multichannel Signal Processing Workshop (SAM '08), pp. 315-319, July 2008.

[13] J. Mather, "The incremental multi-parameter algorithm," in Proceedings of the 24th Asilomar Conference on Signals, Systems \& Computers, vol. 1, pp. 368-372, 1990. 
[14] A. B. Gershman, "Direction finding using beamspace root estimator banks," IEEE Transactions on Signal Processing, vol. 46, no. 11, pp. 3131-3135, 1998.

[15] M. D. Zoltowski, G. M. Kautz, and S. D. Silverstein, "Beamspace root-MUSIC," IEEE Transactions on Signal Processing, vol. 41, no. 1, pp. 344-364, 1993.

[16] L. R. Wyatt, "Progress in the interpretation of HF sea echo: HF radar as a remote sensing tool," IEE Proceedings F, vol. 137, no. 2, pp. 139-147, 1990.

[17] K. W. Gurgel and H. H. Essen, "On the performance of a shipborne current mapping HF radar," IEEE Journal of Oceanic Engineering, vol. 25, no. 1, pp. 183-191, 2000. 

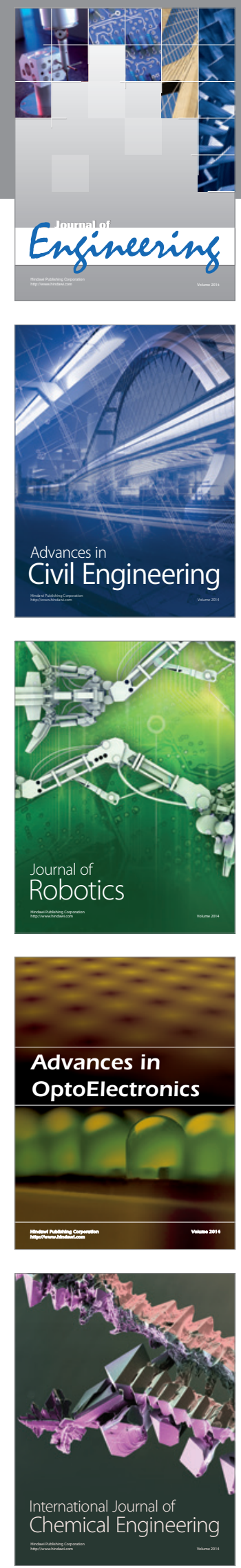

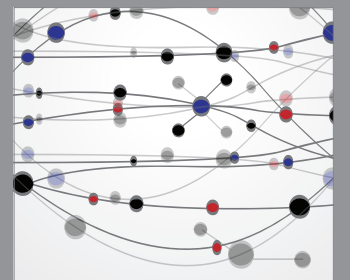

The Scientific World Journal
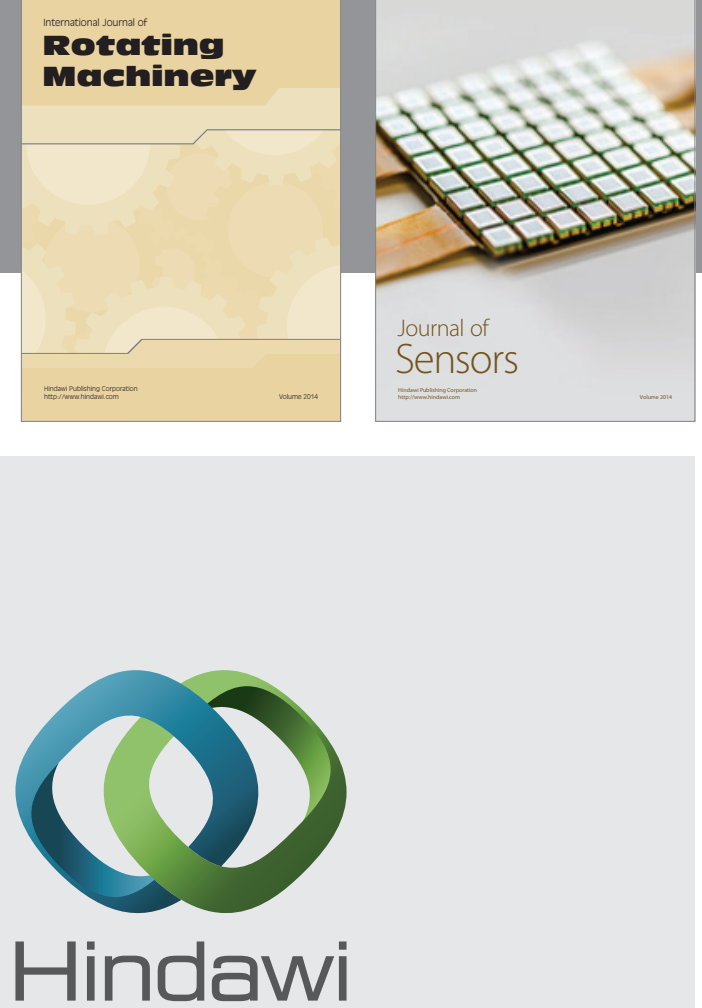

Submit your manuscripts at http://www.hindawi.com
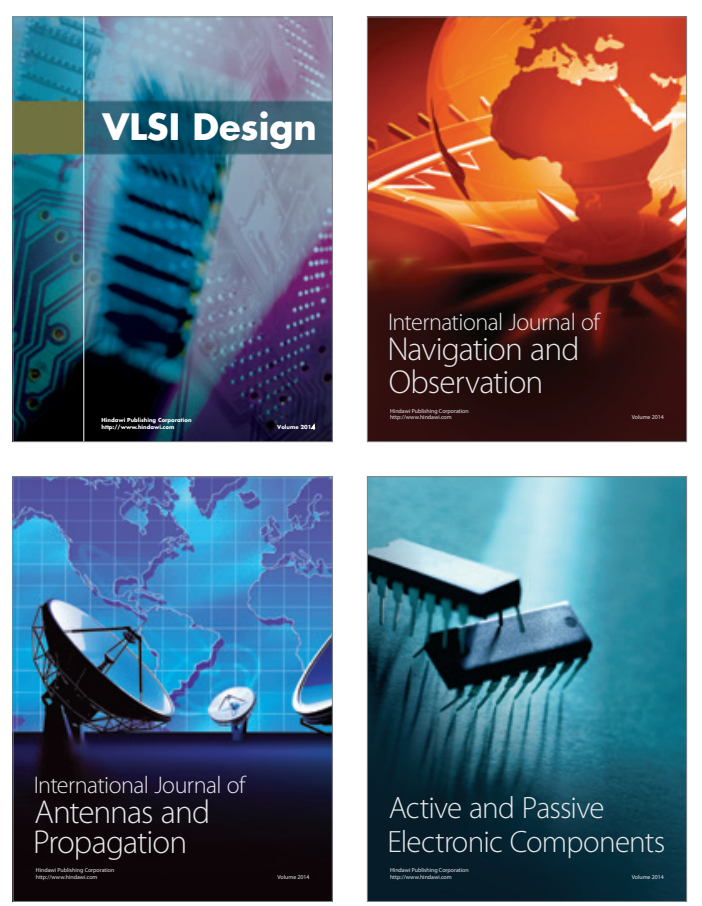
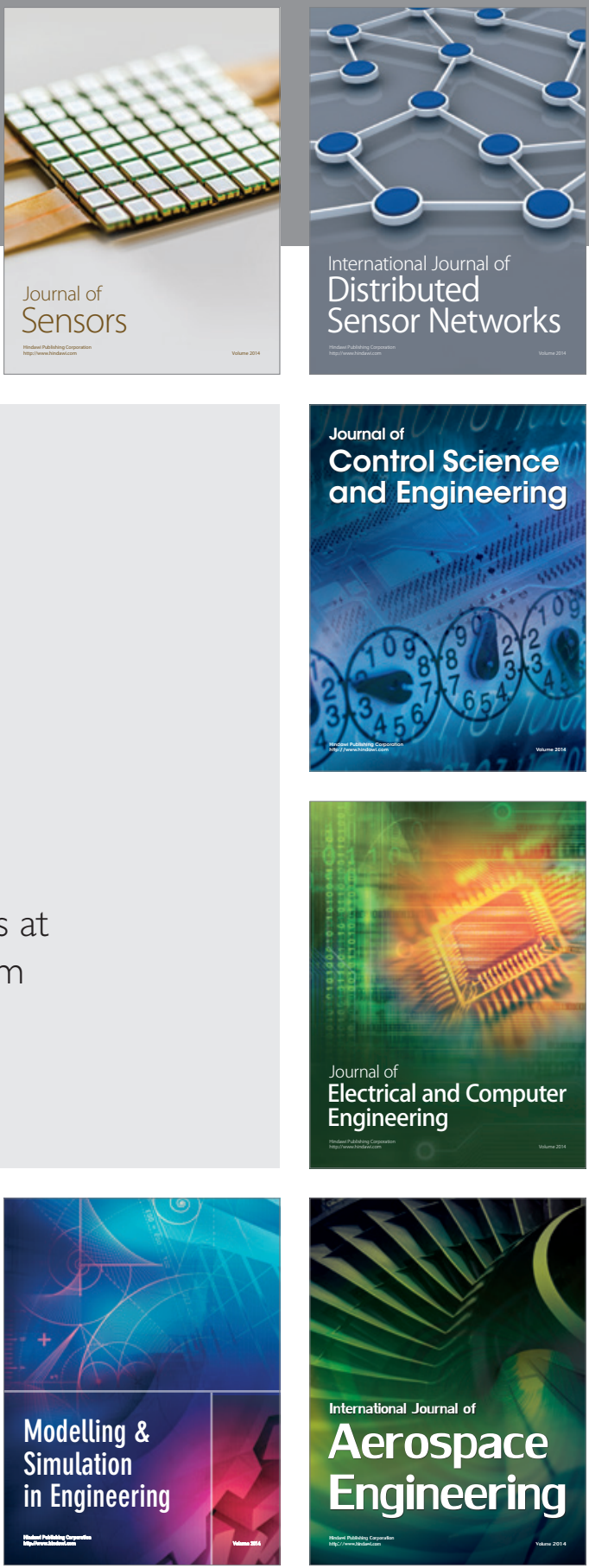

Journal of

Control Science

and Engineering
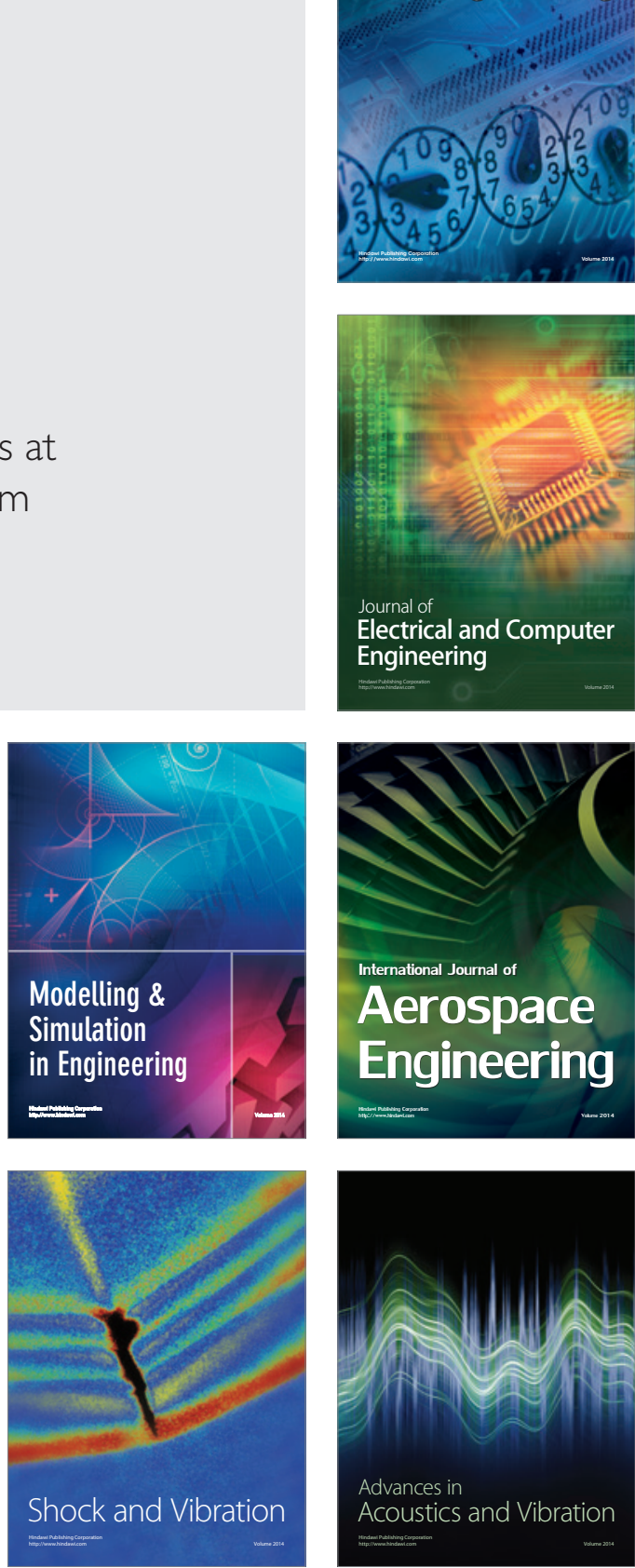